九州大学学術情報リポジトリ

Kyushu University Institutional Repository

\title{
MEAN SQUARED ERRORS OF BOOTSTRAP VARIANCE ESTIMATORS FOR U-STATISTICS
}

Mizuno, Masayuki

Graduate School of Mathematics, School of Mathematics Kyushu University

Maesono, Yoshihiko

Faculty of Mathematics, Kyushu University

https://doi.org/10.5109/1434312

出版情報: Bulletin of informatics and cybernetics. 43，pp.67-82，2011-12. 統計科学研究会 バージョン：

権利関係 : 
MEAN SQUARED ERRORS OF BOOTSTRAP VARIANCE ESTIMATORS FOR $U$-STATISTICS

by

Masayuki Mizuno and Yoshihiko Maesono

Reprinted from the Bulletin of Informatics and Cybernetics Research Association of Statistical Sciences, Vol.43

FUKUOKA, JAPAN

2011 


\title{
MEAN SQUARED ERRORS OF BOOTSTRAP VARIANCE ESTIMATORS FOR $U$-STATISTICS
}

\author{
By \\ Masayuki Mizuno* and Yoshihiko MaEsono $^{\dagger}$
}

\begin{abstract}
In this paper, we obtain an asymptotic representation of the bootstrap variance estimator for a class of $U$-statistics. Using the representation of the estimator, we will obtain a mean squared error of the variance estimator until the order $n^{-2}$. Also we compare the bootstrap and the jackknife variance estimators, theoretically.
\end{abstract}

Key Words and Phrases: U-statistics, Bootstrap, Jackknife, Mean squared error, Variance estimator.

\section{Introduction}

Let $X_{1}, \cdots, X_{n}$ be independently and identically distributed random vectors with distribution function $F(x)$. Let $h\left(x_{1}, \cdots, x_{r}\right)$ be a real valued function which is symmetric in its arguments. For $n \geq r$, let us define a $U$-statistic by

$$
U_{n}=\left(\begin{array}{l}
n \\
r
\end{array}\right)^{-1} \sum_{C_{n, r}} h\left(X_{i_{1}}, \cdots, X_{i_{r}}\right)
$$

where $\sum_{C_{n, r}}$ indicates that the summation is taken over all integers $i_{1}, \cdots, i_{r}$ satisfying $1 \leq i_{n, r}<\cdots<i_{r} \leq n$. $U_{n}$ is a minimum variance unbiased estimator of $\theta=E\left[h\left(X_{1}, \cdots, X_{r}\right)\right]$ and many statistics in common use are members of $U$-statistics or approximated by them.

Several variance estimators for the $U$-statistic are proposed, e.g. Sen's(1960) estimator, jackknife, unbiased, bootstrap etc. Maesono(1998) obtained the asymptotic representations of them and discussed mean squared errors. The jackknife variance estimator $\hat{\sigma}_{J}^{2}$ is given by

$$
\hat{\sigma}_{J}^{2}=\frac{n-1}{n} \sum_{i=1}^{n}\left(U_{n}^{(i)}-U_{n}\right)^{2}
$$

where $U_{n}^{(i)}$ denotes a corresponding $U$-statistic computed from a sample of $n-1$ points with $X_{i}$ left out.

Another competitor is a bootstrap variance estimator. Many papers show that the bootstrap estimator has good properties in most case, and especially, the bootstrap

\footnotetext{
* Graduate School of Mathematics, School of Mathematics, Kyushu University, 744 Motooka, Nishi-ku, Fukuoka 819-0395, Japan.

$\dagger$ Faculty of Mathematics, Kyushu University, Motooka, Fukuoka 819-0395, Japan. tel +81-92-8024480 maesono@math.kyushu-u.ac.jp
} 
variance estimator is superior to the jackknife for the quantile estimator, like the sample median. The theoretical value of the bootstrap estimator is given by

$$
\hat{\sigma}_{B}^{2}=\iint\left[\left(\begin{array}{l}
n \\
r
\end{array}\right)^{-1} \sum_{C_{n, r}} h\left(x_{i_{1}}, \cdots, x_{i_{r}}\right)-\iint h\left(y_{1}, \cdots, y_{r}\right) \prod_{j=1}^{r} d \hat{F}\left(y_{j}\right)\right]^{2} \prod_{i=1}^{n} d \hat{F}\left(x_{i}\right)
$$

where $\hat{F}$ is an estimator of the distribution function $F$. In this paper we consider the case of the empirical distribution

$$
\hat{F}(x)=F_{n}(x)=\frac{1}{n} \sum_{i=1}^{n} I\left(X_{i} \leq x\right)
$$

where $I(\cdot)$ is an indicator function.

Many papers discuss properties of $\hat{\sigma}_{B}^{2}$ by simulations, and show that the bootstrap estimator has the good properties. On the other hand, the properties of $\hat{\sigma}_{J}^{2}$ have been precisely studied. Efron and Stein(1981) have showed that the jackknife variance estimator has positive bias, and the bias reduction for the jackknife variance estimator has been studied by Hinkley(1978), and Efron and Stein(1981). In the case of small sample, using computer simulation, Schucany and Bankson(1989) discussed biases and mean squared errors of the variance estimators. It is easy to see that all above estimators have first order consistency, which means that the normalized estimators converge to the dominant term $r^{2} \xi_{1}^{2}$ of the variance. Shirahata and Sakamoto(1992) have compared several estimators (unbiased estimator, jackknife estimator, bias modified estimator, iterated bootstrap and bootstrap estimators) by computer simulations, and pointed out that the bootstrap variance estimator has smallest mean squared error.

Using the asymptotic representation of the jackknife variance estimator $n \hat{\sigma}_{J}^{2}$ with the residual term $O_{p}\left(n^{-2}\right)$, Maesono(1998) has obtained the mean squared errors until the order $n^{-2}$. In this paper we will obtain an asymptotic representation of the bootstrap variance estimator $n \hat{\sigma}_{B}^{2}$ with residual term $O_{p}\left(n^{-2}\right)$ and discuss a mean squared error.

In Section 2, we will review the Hoeffding decomposition and moment evaluations of the decomposition. In Section 3, we will discuss the asymptotic representations and the mean squared errors of the bootstrap variance estimators, and compare the mean squared errors for the variance and covariance estimation.

\section{Hoeffding decomposition and moment evaluation}

At first we will obtain a representation of $U_{n}$ with a sum of forward martingales. This representation is due to Hoeffding(1961) and plays an important role in the asymptotic theory of $U$-statistics. Under the assumption that $E\left|h\left(X_{1}, \cdots, X_{r}\right)\right|<\infty$, let us define the following notations:

$$
a_{k}\left(x_{1}, \cdots, x_{k}\right)=E\left[h\left(X_{1}, \cdots, X_{r}\right) \mid X_{1}=x_{1}, \cdots, X_{k}=x_{k}\right]-\theta \quad(1 \leq k \leq r),
$$




$$
\begin{aligned}
& g_{1}\left(x_{1}\right)=a_{1}\left(x_{1}\right), \quad g_{2}\left(x_{1}, x_{2}\right)=a_{2}\left(x_{1}, x_{2}\right)-g_{1}\left(x_{1}\right)-g_{1}\left(x_{2}\right), \cdots, \\
& g_{r}\left(x_{1}, \cdots, x_{r}\right)=a_{r}\left(x_{1}, \cdots, x_{r}\right)-\sum_{j=1}^{r-1} \sum_{C_{r, j}} g_{j}\left(x_{i_{1}}, \cdots, x_{i_{j}}\right), \\
& A_{k}=\sum_{C_{n, k}} g_{k}\left(X_{i_{1}}, \cdots, X_{i_{k}}\right), \quad \sigma_{n}^{2}=\operatorname{Var}\left(U_{n}\right), \\
& \xi_{1}^{2}=E g_{1}^{2}\left(X_{1}\right), \quad \xi_{2}^{2}=E\left[g_{2}^{2}\left(X_{1}, X_{2}\right)\right] .
\end{aligned}
$$

Then we have

$$
U_{n}-E\left(U_{n}\right)=\left(\begin{array}{c}
n \\
r
\end{array}\right)^{-1} \sum_{k=1}^{r}\left(\begin{array}{c}
n-k \\
r-k
\end{array}\right) A_{k}
$$

and

$$
E\left[g_{k}\left(X_{1}, \cdots, X_{k}\right) \mid X_{1}, \cdots, X_{k-1}\right]=0 \text { a.s. }
$$

Note that $g_{k}(1 \leq k \leq r)$ are orthogonal in the sense that their covariances are zero, and $\left\{A_{k}\right\}_{n \geq k}$ is a forward martingale for $k=1, \cdots, r$. This method is called as $H$ decomposition or $A N O V A$ - decomposition, and is familiar in the studies of the analysis of variance, the jackknife inference, and so on. Using the equation (1) we have the variance $\sigma_{n}^{2}$ of $U_{n}$

$$
\begin{aligned}
\sigma_{n}^{2} & =\operatorname{Var}\left(U_{n}\right)=\sum_{k=1}^{r}\left(\begin{array}{c}
r \\
k
\end{array}\right)^{2}\left(\begin{array}{c}
n \\
k
\end{array}\right)^{-1} E\left[g_{k}^{2}\left(X_{1}, \cdots, X_{k}\right)\right] \\
& =\frac{r^{2}}{n} \xi_{1}^{2}+\frac{[r(r-1)]^{2}}{2 n(n-1)} \xi_{2}^{2}+\cdots+\frac{r !}{n(n-1) \cdots(n-r+1)} E\left[g_{r}^{2}\left(X_{1}, \cdots, X_{r}\right)\right] .
\end{aligned}
$$

From von Bahr and Esséen(1965) and Dharmadhikari, Fabian and Jogdeo(1968), we have the upper bounds of the absolute moments of $A_{k}$ as follows. Since we will use moment evaluations for another kernel, we state the Lemma in general form.

Lemma 2.1. Let $\rho\left(x_{1}, x_{2}, \cdots, x_{k}\right)$ be a real valued function which is symmetric in its arguments. Assume that $E\left[\rho\left(X_{1}, X_{2}, \cdots, X_{k}\right) \mid X_{1}, X_{2}, \cdots, X_{k-1}\right]=0$ a.s. Then we have the following inequalities.

(i) For $1 \leq p<2$, if $E\left|\rho\left(X_{1}, \cdots, X_{k}\right)\right|^{p}<\infty$, there exists a positive constant $c_{h}$, which may depend on $\rho$ and $F$ but not on $n$, such that

$$
E\left|\sum_{C_{n, k}} \rho\left(X_{i_{1}}, \cdots, X_{i_{k}}\right)\right|^{p} \leq c_{h} n^{k} .
$$

(ii) For $2 \leq p$, if $E\left|\rho\left(X_{1}, \cdots, X_{k}\right)\right|^{p}<\infty$, there exists a positive constant $c_{h}^{\prime}$, which may depend on $\rho$ and $F$ but not on $n$, such that

$$
E\left|\sum_{C_{n, k}} \rho\left(X_{i_{1}}, \cdots, X_{i_{k}}\right)\right|^{p} \leq c_{h}^{\prime} n^{p k / 2} .
$$

Proof. See Maesono(1998). 
Hereafter for the sake of simplicity, we will consider the kernel of degree $r=2$. The generalization to the kernel of arbitrary degree will be obtained with notational complications and tedious calculations. We consider the bootstrap variance estimator $\hat{\sigma}_{B}^{2}$ for the $U$-statistic

$$
\hat{\sigma}_{B}^{2}=\iint\left[\left(\begin{array}{l}
n \\
2
\end{array}\right)^{-1} \sum_{C_{n, 2}} h\left(x_{i}, x_{j}\right)-\iint h\left(y_{1}, y_{2}\right) d F_{n}\left(y_{1}\right) d F_{n}\left(y_{2}\right)\right]^{2} \prod_{i=1}^{n} d F_{n}\left(x_{k}\right) .
$$

For the kernel $h(x, y)$, we have

$$
\begin{aligned}
& g_{1}(x)=E\left[h\left(x, X_{2}\right)\right]-\theta, \quad g_{2}(x, y)=h(x, y)-\theta-g_{1}(x)-g_{1}(y), \\
& A_{1}=\sum_{i=1}^{n} g_{1}\left(X_{i}\right), \quad A_{2}=\sum_{C_{n, 2}} g_{2}\left(X_{i}, X_{j}\right)
\end{aligned}
$$

and

$$
U_{n}-\theta=\frac{2}{n} A_{1}+\frac{2}{n(n-1)} A_{2}
$$

Note that

$$
E\left[g_{2}\left(X_{1}, X_{2}\right) \mid X_{1}\right]=0 \text { a.s. }
$$

Thus if one of $\left\{i_{1}, i_{2}\right\}$ is not contained in $\left\{j_{1}, \cdots, j_{m}\right\}$, for any $m$-variate function $\nu$ which satisfies $E\left|\nu g_{2}\right|<\infty$, we get

$$
E\left[g_{k}\left(X_{i_{1}}, X_{i_{2}}\right) \nu\left(X_{j_{1}}, \cdots, X_{j_{m}}\right)\right]=0
$$

Using this equation we have the variance $\sigma_{n}^{2}$ of $U_{n}$

$$
\sigma_{n}^{2}=\frac{4}{n} \xi_{1}^{2}+\frac{2}{n(n-1)} \xi_{2}^{2}
$$

where

$$
\xi_{1}^{2}=E\left[g_{1}^{2}\left(X_{1}\right)\right] \quad \text { and } \quad \xi_{2}^{2}=E\left[g_{2}^{2}\left(X_{1}, X_{2}\right)\right]
$$

Since we discuss the asymptotic properties of the variance estimators, we will study the estimation of $n \sigma_{n}^{2}$. Thus we consider the bootstrap variance estimator $V_{B}=n \hat{\sigma}_{B}^{2}$ and the jackknife estimator $V_{J}=n \hat{\sigma}_{J}^{2}$. 


\section{Asymptotic representations and mean squared errors}

For the variance estimators $V_{J}$, Maesono(1998) has obtained the asymptotic representations with residual terms $O_{p}\left(n^{-2}\right)$. Let us define

$$
\begin{aligned}
h^{*}(x, y)= & h(x, y)-\theta \\
\delta(x)= & E\left[g_{2}^{2}\left(x, X_{2}\right)\right]-\xi_{2}^{2}, \\
\delta^{*}(x)= & -16 E\left[h^{*}\left(X_{2}, X_{2}\right) h^{*}\left(x, X_{3}\right)\right]+8 E\left[h^{*}(x, x) h^{*}\left(x, X_{2}\right)\right] \\
& +8 E\left[h^{*}\left(x, X_{2}\right) h^{*}\left(X_{2}, X_{2}\right)\right]-16 E\left[h^{*}\left(X_{1}, X_{1}\right) h^{*}\left(X_{1}, X_{2}\right)\right], \\
f_{1}(x)= & g_{1}^{2}(x)-\xi_{1}^{2}+2 E\left[g_{1}\left(X_{2}\right) g_{2}\left(x, X_{2}\right)\right], \\
f_{2}(x, y)= & -g_{1}(x) g_{1}(y)+g_{2}(x, y)\left\{g_{1}(x)+g_{1}(y)\right\} \\
& +E\left[g_{2}\left(x, X_{3}\right) g_{2}\left(y, X_{3}\right)-g_{2}\left(x, X_{3}\right) g_{1}\left(X_{3}\right)-g_{2}\left(y, X_{3}\right) g_{1}\left(X_{3}\right)\right], \\
f_{3}(x, y, z)= & g_{2}(x, y) g_{2}(x, z)+g_{2}(x, y) g_{2}(y, z)+g_{2}(x, z) g_{2}(y, z) \\
- & E\left[g_{2}\left(x, X_{3}\right) g_{2}\left(y, X_{3}\right)+g_{2}\left(y, X_{3}\right) g_{2}\left(z, X_{3}\right)+g_{2}\left(x, X_{3}\right) g_{2}\left(z, X_{3}\right)\right] \\
- & 2\left\{g_{1}(x) g_{2}(y, z)+g_{1}(y) g_{2}(x, z)+g_{1}(z) g_{2}(x, y)\right\}
\end{aligned}
$$

and

$$
V_{n}=\frac{4}{n} \sum_{i=1}^{n} f_{1}\left(X_{i}\right)+\frac{8}{n^{2}} \sum_{C_{n, 2}} f_{2}\left(X_{i}, X_{j}\right)+\frac{8}{n^{3}} \sum_{C_{n, 3}} f_{3}\left(X_{i}, X_{j}, X_{k}\right) .
$$

Note that $V_{n}$ has already decomposed. For the jackknife variance estimator, Maesono (1998) obtained the following asymptotic representation

$$
V_{J}=V_{n}+\frac{8}{n^{2}} \sum_{i=1}^{n} \delta\left(X_{i}\right)+n \sigma_{n}^{2}+\frac{1}{n} b_{J}+R_{1 ; n}
$$

where

$$
b_{J}=2 \xi_{2}^{2}
$$

and $E\left|R_{1 ; n}\right|^{2+\varepsilon / 2}=O\left(n^{-4-\varepsilon}\right)$ for some $\varepsilon>0$. Similarly to $V_{J}$, we have an asymptotic representation of $V_{B}$ as follows.

Theorem 3.1. If $E\left|h\left(X_{i}, X_{j}\right)\right|^{4+\varepsilon}<\infty(i, j=1,2)$ for some $\varepsilon>0$, we have

$$
V_{B}=V_{n}+\frac{1}{n^{2}} \sum_{i=1}^{n}\left\{12 \delta\left(X_{i}\right)-20 f_{1}\left(X_{i}\right)+\delta^{*}\left(X_{i}\right)\right\}+n \sigma_{n}^{2}+\frac{1}{n} b_{B}+R_{2 ; n}
$$

where

$$
b_{B}=4 \xi_{2}^{2}-20 \xi_{1}^{2}+8 E\left[h^{*}\left(X_{1}, X_{1}\right) h^{*}\left(X_{1}, X_{2}\right)\right]
$$

and $E\left|R_{2 ; n}\right|^{2+\varepsilon / 2}=O\left(n^{-4-\varepsilon}\right)$. 
Proof. See appendix.

$b_{J}$ and $b_{B}$ are $n^{-1}$ biases of the jackknife and the bootstrap variance estimators, respectively. It is easy to see that $E\left[f_{1}\left(X_{1}\right)\right]=E\left[\delta\left(X_{1}\right)\right]=E\left[\delta^{*}\left(X_{1}\right)\right]=0$ and

$$
E\left[f_{2}\left(X_{1}, X_{2}\right) \mid X_{1}\right]=E\left[f_{3}\left(X_{1}, X_{2}, X_{3}\right) \mid X_{1}, X_{2}\right]=0 \text { a.s. }
$$

Using the asymptotic representations of Theorem 3.1, we can study the asymptotic properties of the variance estimators. Here we will obtain mean squared errors of $V_{J}$ and $V_{B}$ up to the order $n^{-2}$. Let us define

$$
\begin{aligned}
\operatorname{mse}\left(V_{J}\right) & =\frac{16}{n} E\left[f_{1}^{2}\left(X_{1}\right)\right] \\
& +\frac{1}{n^{2}}\left\{b_{J}^{2}+64 E\left[f_{1}\left(X_{1}\right) \delta\left(X_{1}\right)\right]+32 E\left[f_{2}^{2}\left(X_{1}, X_{2}\right)\right]\right\}
\end{aligned}
$$

and

$$
\begin{aligned}
\operatorname{mse}\left(V_{B}\right)= & \frac{16}{n} E\left[f_{1}^{2}\left(X_{1}\right)\right]+\frac{1}{n^{2}}\left\{b_{B}^{2}+96 E\left[f_{1}\left(X_{1}\right) \delta\left(X_{1}\right)\right]\right. \\
& \left.-160 E\left[f_{1}^{2}\left(X_{1}\right)\right]+8 E\left[f_{1}\left(X_{1}\right) \delta^{*}\left(X_{1}\right)\right]+32 E\left[f_{2}^{2}\left(X_{1}, X_{2}\right)\right]\right\} .
\end{aligned}
$$

Then Maesono(1998) showed that

$$
E\left(V_{J}-n \sigma_{n}^{2}\right)^{2}=m s e\left(V_{J}\right)+O\left(n^{-5 / 2}\right) .
$$

For the bootstrap estimator $V_{B}$, we have the following theorem.

Theorem 3.2. If $E\left|h\left(X_{i}, X_{j}\right)\right|^{4+\varepsilon}<\infty(i, j=1,2)$ for some $\varepsilon>0$, we have

$$
E\left(V_{B}-n \sigma_{n}^{2}\right)^{2}=m s e\left(V_{B}\right)+O\left(n^{-5 / 2}\right) .
$$

Proof. It follows from Theorem 3.1 that under the moment condition, for $1 \leq$ $k \leq 5$,

$$
\begin{aligned}
E\left|n^{-1} R_{2 ; n} \sum_{i=1}^{n} f_{1}\left(X_{i}\right)\right| & \leq n^{-1}\left\{E\left|\sum_{i=1}^{n} f_{1}\left(X_{i}\right)\right|^{2+\varepsilon / 2} E\left|R_{2 ; n}\right|^{2+\varepsilon / 2}\right\}^{2 /(4+\varepsilon)} \\
& =O\left(n^{-5 / 2}\right), \\
E\left|n^{-2} R_{2 ; n} \sum_{i=1}^{n} \delta\left(X_{i}\right)\right| & \leq n^{-2}\left\{E\left|\sum_{i=1}^{n} \delta\left(X_{i}\right)\right|^{2+\varepsilon / 2} E\left|R_{2 ; n}\right|^{2+\varepsilon / 2}\right\}^{2 /(4+\varepsilon)} \\
& =O\left(n^{-3}\right), \\
E\left|n^{-2} R_{2 ; n} \sum_{C_{n, 2}} f_{2}\left(X_{i}, X_{j}\right)\right| & \leq n^{-2}\left\{E\left|\sum_{C_{n, 2}} f_{2}\left(X_{i}, X_{j}\right)\right|^{2+\varepsilon / 2} E\left|R_{2 ; n}\right|^{2+\varepsilon / 2}\right\}^{2 /(4+\varepsilon)} \\
& =O\left(n^{-3}\right)
\end{aligned}
$$

and

$$
E\left|R_{2 ; n}\right|^{2} \leq\left\{E\left|R_{2 ; n}\right|^{2+\varepsilon / 2}\right\}^{4 /(4+\varepsilon)}=O\left(n^{-4}\right) .
$$

Thus, using these equations and (9), we can obtain the desired result. 
REMARK. It is possible to improve the equations with remainder terms of the order $O\left(n^{-3}\right)$. But it needs more calculation, then we leave the equation as it is.

Let us define

$$
\begin{aligned}
e_{1} & =E\left[g_{1}^{4}\left(X_{1}\right)\right], \quad e_{2}=E\left[g_{1}^{2}\left(X_{1}\right) g_{2}^{2}\left(X_{1}, X_{2}\right)\right], \\
e_{3} & =E\left[g_{1}\left(X_{1}\right) g_{2}\left(X_{1}, X_{2}\right) g_{2}^{2}\left(X_{2}, X_{3}\right)\right], \\
e_{4} & =E\left[g_{1}^{2}\left(X_{1}\right) g_{1}\left(X_{2}\right) g_{2}\left(X_{1}, X_{2}\right)\right], \\
e_{5} & =E\left[g_{1}\left(X_{1}\right) g_{1}\left(X_{2}\right) g_{2}^{2}\left(X_{1}, X_{2}\right)\right], \\
e_{6} & =E\left[g_{1}\left(X_{1}\right) g_{1}\left(X_{2}\right) g_{2}\left(X_{1}, X_{3}\right) g_{2}\left(X_{2}, X_{3}\right)\right], \\
e_{7} & =E\left[g_{1}\left(X_{1}\right) g_{2}\left(X_{1}, X_{2}\right) g_{2}\left(X_{1}, X_{3}\right) g_{2}\left(X_{2}, X_{3}\right)\right]
\end{aligned}
$$

and

$$
e_{8}=E\left[g_{2}\left(X_{1}, X_{3}\right) g_{2}\left(X_{2}, X_{3}\right) g_{2}\left(X_{1}, X_{4}\right) g_{2}\left(X_{2}, X_{4}\right)\right] \text {. }
$$

Then, using the equation (1), it follows from direct computations that

$$
\begin{aligned}
& E\left[f_{1}^{2}\left(X_{1}\right)\right]=e_{1}-\xi_{1}^{4}+4 e_{4}+4 e_{6} \\
& E\left[f_{1}\left(X_{1}\right) \delta\left(X_{1}\right)\right]=e_{2}-\xi_{1}^{2} \xi_{2}^{2}+2 e_{3}
\end{aligned}
$$

and

$$
E\left[f_{2}^{2}\left(X_{1}, X_{2}\right)\right]=\xi_{1}^{4}+2 e_{2}-4 e_{4}+2 e_{5}-4 e_{6}+4 e_{7}+e_{8}
$$

Here we will study the mean squared errors for the variance and the covariance estimation problems.

EXAMPLE 3.3. Variance estimation;

Let us consider the kernel $h(x, y)=(x-y)^{2} / 2$. Then if $E\left(X_{1}\right)=\mu$ and $\operatorname{Var}\left(X_{1}\right)=\sigma^{2}$ exist, the $U$-statistic

$$
U_{n}=\left(\begin{array}{l}
n \\
2
\end{array}\right)^{-1} \sum_{C_{n, 2}} h\left(X_{i}, X_{j}\right)
$$

is an unbiased estimator of $\sigma^{2}$. Note that $h\left(x_{1}, x_{1}\right)=0$ and then $\delta^{*}(x)=0$. It is easy to see that

$$
\theta=\sigma^{2}, \quad g_{1}(x)=\frac{1}{2}\left\{(x-\mu)^{2}-\sigma^{2}\right\} \quad \text { and } \quad g_{2}(x, y)=-(x-\mu)(y-\mu) .
$$

For the sake of simplicity, we will consider the case that the distribution $F(x)$ is symmetric about the mean $\mu$. Let us define $m_{k}=E\left\{\left(X_{1}-\mu\right)^{k}\right\}$. Then because of symmetry of $F$, if $k$ is odd number, $m_{k}=0$. Using this fact, it follows from direct computations that

$$
\begin{aligned}
& \xi_{1}^{2}=\frac{1}{4}\left(m_{4}-\sigma^{4}\right), \quad \xi_{2}^{2}=\sigma^{4}, \quad e_{1}=\frac{1}{16}\left(m_{8}-4 \sigma^{2} m_{6}+6 \sigma^{4} m_{4}-3 \sigma^{8}\right), \\
& e_{2}=\frac{\sigma^{2}}{4}\left(m_{6}-2 \sigma^{2} m_{4}+\sigma^{6}\right), \quad e_{5}=\frac{1}{4}\left(m_{4}-\sigma^{4}\right)^{2}, \quad e_{7}=-\frac{\sigma^{4}}{2}\left(m_{4}-\sigma^{4}\right), \\
& e_{8}=\sigma^{8} \text { and } e_{3}=e_{4}=e_{6}=0 .
\end{aligned}
$$


[Normal distribution:] If the underlying distribution is normal, that is $X_{i} \sim N\left(\mu, \sigma^{2}\right)$, we can show that

$$
b_{J}=2 \sigma^{4} \quad \text { and } \quad b_{B}=-6 \sigma^{4} .
$$

Then we have the mean squared errors

$$
m s e\left(V_{J}\right)=\sigma^{8}\left\{\frac{56}{n}+\frac{268}{n^{2}}\right\} \text { and } m s e\left(V_{B}\right)=\sigma^{8}\left\{\frac{56}{n}-\frac{196}{n^{2}}\right\}
$$

In the case of $\sigma^{2}=1$ and $n=10$, Schucany and Bankson(1989) discussed the mean squared error of $V_{J} / n$, by simulation. Corresponding asymptotic mean squared error is given by

$$
\frac{m s e\left(V_{J}\right)}{10^{2}}=0.0828 \text {. }
$$

Their estimated mean squared error is close to this value.

[Logistic distribution:] We consider the logistic distribution which has the density function

$$
\frac{\pi e^{-\frac{\pi x}{\sqrt{3} \sigma}}}{\sqrt{3} \sigma\left(1+e^{-\frac{\pi x}{\sqrt{3} \sigma}}\right)} .
$$

In this case we have that $\operatorname{Var}\left(X_{1}\right)=\sigma^{2}$,

$$
\begin{aligned}
& b_{J}=2 \sigma^{4}, \quad b_{B}=-8 \sigma^{4}, \\
& m s e\left(V_{J}\right)=\sigma^{8}\left\{\frac{538.33}{n}+\frac{1002.95}{n^{2}}\right\}
\end{aligned}
$$

and

$$
m s e\left(V_{B}\right)=\sigma^{8}\left\{\frac{538.33}{n}-\frac{4086.31}{n^{2}}\right\} .
$$

EXAMPLE 3.4. Covariance estimation;

Let $\left\{\boldsymbol{X}_{i}\right\}_{i \geq 1}$ be two dimensional random vectors, and putting $\boldsymbol{X}_{i}=\left(Y_{i}, Z_{i}\right)$, we denote

$$
\operatorname{Var}\left(\boldsymbol{X}_{1}\right)=\operatorname{Var}\left\{\left(Y_{1}, Z_{1}\right)\right\}=\left(\begin{array}{cc}
\sigma_{y}^{2} & \rho \sigma_{y} \sigma_{z} \\
\rho \sigma_{y} \sigma_{z} & \sigma_{z}^{2}
\end{array}\right) .
$$

Let us consider a symmetric kernel $h\left(\boldsymbol{x}_{1}, \boldsymbol{x}_{2}\right)=\left(y_{1}-y_{2}\right)\left(z_{1}-z_{2}\right) / 2$. Then corresponding $U$-statistic is an unbiased estimator of $\rho \sigma_{y} \sigma_{z}=\operatorname{Cov}\left(Y_{1}, Z_{1}\right)$. It is easy to see that

$$
\theta=\rho \sigma_{y} \sigma_{z}, \quad g_{1}\left(\boldsymbol{x}_{1}\right)=\frac{1}{2}\left\{\left(y_{1}-\mu_{y}\right)\left(z_{1}-\mu_{z}\right)-\rho \sigma_{y} \sigma_{z}\right\}
$$

and

$$
g_{2}\left(\boldsymbol{x}_{1}, \boldsymbol{x}_{2}\right)=-\frac{1}{2}\left\{\left(y_{1}-\mu_{y}\right)\left(z_{2}-\mu_{z}\right)+\left(z_{1}-\mu_{z}\right)\left(y_{2}-\mu_{y}\right)\right\}
$$

where $\mu_{y}=E\left(Y_{1}\right)$ and $\mu_{z}=E\left(Z_{1}\right)$. Note that $h\left(\boldsymbol{x}_{1}, \boldsymbol{x}_{2}\right)=0$. Here we assume that $\boldsymbol{X}_{i}$ is the bivariate normal distribution

$$
\boldsymbol{X}_{i}=\left(Y_{i}, Z_{i}\right) \sim N\left(\left(\begin{array}{c}
\mu_{y} \\
\mu_{z}
\end{array}\right),\left(\begin{array}{cc}
\sigma_{y}^{2} & \rho \sigma_{y} \sigma_{z} \\
\rho \sigma_{y} \sigma_{z} & \sigma_{z}^{2}
\end{array}\right)\right) .
$$


From direct computations we can get

$$
\begin{aligned}
& \xi_{1}^{2}=\frac{1+\rho^{2}}{4} \sigma_{y}^{2} \sigma_{z}^{2}, \quad \xi_{2}^{2}=\frac{1+\rho^{2}}{2} \sigma_{y}^{2} \sigma_{z}^{2}, \quad e_{1}=\frac{3}{16}\left(3 \rho^{4}+14 \rho^{2}+3\right) \sigma_{y}^{4} \sigma_{z}^{4} \\
& e_{2}=\frac{1}{8}\left(3 \rho^{4}+14 \rho^{2}+3\right) \sigma_{y}^{4} \sigma_{z}^{4}, \quad e_{3}=e_{4}=0, \quad e_{5}=\frac{1}{8}\left(\rho^{4}+6 \rho^{2}+1\right) \sigma_{y}^{4} \sigma_{z}^{4} \\
& e_{6}=0, \quad e_{7}=-\frac{1}{8}\left(\rho^{4}+6 \rho^{2}+1\right) \sigma_{y}^{4} \sigma_{z}^{4} \quad \text { and } \quad e_{8}=\frac{1}{8}\left(\rho^{4}+6 \rho^{2}+1\right) \sigma_{y}^{4} \sigma_{z}^{4} .
\end{aligned}
$$

Thus we have

$$
\begin{aligned}
& b_{J}=\sigma_{y}^{2} \sigma_{z}^{2}\left(1+\rho^{2}\right), \quad b_{B}=-3\left(1+\rho^{2}\right) \sigma_{y}^{2} \sigma_{z}^{2} \\
& m s e\left(V_{J}\right)=\sigma_{y}^{4} \sigma_{z}^{4}\left\{\frac{8}{n}\left(\rho^{4}+5 \rho^{2}+1\right)+\frac{1}{n^{2}}\left(39 \rho^{4}+190 \rho^{2}+39\right)\right\}
\end{aligned}
$$

and

$$
m s e\left(V_{B}\right)==\sigma_{y}^{4} \sigma_{z}^{4}\left\{\frac{8}{n}\left(\rho^{4}+5 \rho^{2}+1\right)-\frac{1}{n^{2}}\left(25 \rho^{4}+146 \rho^{2}+25\right)\right\}
$$

REMARK. In the cases of the above tow examples, $m s e\left(V_{B}\right)<m s e\left(V_{J}\right)$. We had better to study the properties of both estimators more precisely.

\section{Appendix}

Proof of Theorem 1 From the definition, we have

$$
\begin{gathered}
\hat{\sigma}_{B}^{2}=\iint\left[\left(\begin{array}{l}
n \\
2
\end{array}\right)^{-1} \sum_{C_{n, 2}} h\left(x_{i}, x_{j}\right)-\iint h\left(y_{1}, y_{2}\right) d F_{n}\left(y_{1}\right) d F_{n}\left(y_{2}\right)\right]^{2} \prod_{k=1}^{n} d F_{n}\left(x_{k}\right) \\
=\iint\left(\begin{array}{l}
n \\
2
\end{array}\right)^{-2}\left[\sum_{C_{n, 2}} h^{*}\left(x_{i}, x_{j}\right)\right]^{2} \prod_{k=1}^{n} d F_{n}\left(x_{k}\right) \\
-\left[\iint h^{*}\left(y_{1}, y_{2}\right) d F_{n}\left(y_{1}\right) d F_{n}\left(y_{2}\right)\right]^{2} .
\end{gathered}
$$

For the first term of (12), it is easy to get the following equation

$$
\begin{aligned}
& \iint\left(\begin{array}{l}
n \\
2
\end{array}\right)^{-2}\left[\sum_{C_{n, 2}} h^{*}\left(x_{i}, x_{j}\right)\right]^{2} \prod_{k=1}^{n} d F_{n}\left(x_{k}\right) \\
= & \frac{1}{n^{2}(n-1)^{2}} \iint\left(\sum_{i \neq j} h^{*}\left(x_{i}, x_{j}\right)\right)^{2} \prod_{k=1}^{n} d F_{n}\left(x_{k}\right) \\
= & \frac{1}{n^{2}(n-1)^{2}} \iint \sum_{i \neq j} \sum_{\ell \neq m} h^{*}\left(x_{i}, x_{j}\right) h^{*}\left(x_{\ell}, x_{m}\right) \prod_{k=1}^{n} d F_{n}\left(x_{k}\right) .
\end{aligned}
$$


Let us define

$$
\begin{aligned}
& \zeta_{0}\left(x_{1}, x_{2}, x_{3}, x_{4}\right) \\
= & \frac{1}{3}\left\{h^{*}\left(x_{1}, x_{2}\right) h^{*}\left(x_{3}, x_{4}\right)+h^{*}\left(x_{1}, x_{3}\right) h^{*}\left(x_{2}, x_{4}\right)+h^{*}\left(x_{1}, x_{4}\right) h^{*}\left(x_{2}, x_{3}\right)\right\}, \\
& \zeta_{1}\left(x_{1}, x_{2}, x_{3}\right) \\
= & \frac{1}{3}\left\{h^{*}\left(x_{1}, x_{2}\right) h^{*}\left(x_{1}, x_{3}\right)+h^{*}\left(x_{1}, x_{2}\right) h^{*}\left(x_{2}, x_{3}\right)+h^{*}\left(x_{1}, x_{3}\right) h^{*}\left(x_{2}, x_{3}\right)\right\}
\end{aligned}
$$

and $\zeta_{2}\left(x_{1}, x_{2}\right)=\left\{h^{*}\left(x_{1}, x_{2}\right)\right\}^{2}$. Then for the first term of (12), we have

$$
\begin{aligned}
& \iint\left(\begin{array}{l}
n \\
2
\end{array}\right)^{-2}\left[\sum_{C_{n, 2}} h^{*}\left(x_{i}, x_{j}\right)\right]^{2} \prod_{k=1}^{n} d F_{n}\left(x_{k}\right) \\
= & \frac{1}{n^{2}(n-1)^{2}} \int \cdots \int\left(2 n(n-1) \zeta_{2}\left(x_{1}, x_{2}\right)+4 n(n-1)(n-2) \zeta_{1}\left(x_{1}, x_{2}, x_{3}\right)\right. \\
& \left.+n(n-1)(n-2)(n-3) \zeta_{0}\left(x_{1}, x_{2}, x_{3}, x_{4}\right)\right) \prod_{k=1}^{4} d F_{n}\left(x_{k}\right) \\
= & \frac{2}{n^{3}(n-1)} \sum_{i=1}^{n} \sum_{j=1}^{n} \zeta_{2}\left(X_{i}, X_{j}\right)+\frac{4(n-2)}{n^{4}(n-1)} \sum_{i=1}^{n} \cdots \sum_{k=1}^{n} \zeta_{1}\left(X_{i}, X_{j}, X_{k}\right) \\
+ & \frac{(n-2)(n-3)}{n^{5}(n-1)} \sum_{i=1}^{n} \cdots \sum_{\ell=1}^{n} \zeta_{0}\left(X_{i}, X_{j}, X_{k}, X_{\ell}\right) .
\end{aligned}
$$

Furthermore, for each $\zeta_{k}$ we can show that

$$
\begin{aligned}
& \sum_{i=1}^{n} \sum_{j=1}^{n} \zeta_{2}\left(X_{i}, X_{j}\right) \\
= & \sum_{i=1}^{n}\left\{h^{*}\left(X_{i}, X_{i}\right)\right\}^{2}+2 \sum_{C_{n, 2}} \zeta_{2}\left(X_{i}, X_{j}\right), \\
& \sum_{i=1}^{n} \cdots \sum_{k=1}^{n} \zeta_{1}\left(X_{i}, X_{j}, X_{k}\right) \\
= & \sum_{i=1}^{n}\left\{h^{*}\left(X_{i}, X_{i}\right)\right\}^{2}+2 \sum_{C_{n, 2}} \zeta_{2}\left(X_{i}, X_{j}\right) \\
& +2 \sum_{C_{n, 2}}\left\{h^{*}\left(X_{i}, X_{i}\right) h^{*}\left(X_{i}, X_{j}\right)+h^{*}\left(X_{i}, X_{j}\right) h^{*}\left(X_{j}, X_{j}\right)\right\} \\
& +6 \sum_{C_{n, 3}} \zeta_{1}\left(X_{i}, X_{j}, X_{k}\right)
\end{aligned}
$$


and

$$
\begin{aligned}
& \sum_{i=1}^{n} \cdots \sum_{l=1}^{n} \zeta_{0}\left(X_{i}, X_{j}, X_{k}, X_{l}\right) \\
= & \sum_{i=1}^{n}\left\{h^{*}\left(X_{i}, X_{i}\right)\right\}^{2}+4 \sum_{C_{n, 2}} \zeta_{2}\left(X_{i}, X_{j}\right) \\
& +4 \sum_{C_{n, 2}}\left\{h^{*}\left(X_{i}, X_{i}\right) h^{*}\left(X_{i}, X_{j}\right)+h^{*}\left(X_{i}, X_{j}\right) h^{*}\left(X_{j}, X_{j}\right)\right\} \\
& +2 \sum_{C_{n, 2}} h^{*}\left(X_{i}, X_{i}\right) h^{*}\left(X_{j}, X_{j}\right)+24 \sum_{C_{n, 3}} \zeta_{1}\left(X_{i}, X_{j}, X_{k}\right) \\
& +4 \sum_{C_{n, 3}}\left\{h^{*}\left(X_{i}, X_{i}\right) h^{*}\left(X_{j}, X_{k}\right)+h^{*}\left(X_{j}, X_{j}\right) h^{*}\left(X_{i}, X_{k}\right)+h^{*}\left(X_{k}, X_{k}\right) h^{*}\left(X_{i}, X_{j}\right)\right\} \\
+ & 24 \sum_{C_{n, 4}} \zeta_{0}\left(X_{i}, X_{j}, X_{k}, X_{\ell}\right) .
\end{aligned}
$$

Substituting these terms, we get

$$
\begin{aligned}
& \iint\left(\begin{array}{l}
n \\
2
\end{array}\right)^{-2}\left[\sum_{C_{n, 2}} h^{*}\left(x_{i}, x_{j}\right)\right]^{2} \prod_{k=1}^{n} d F_{n}\left(x_{k}\right) \\
= & \frac{7 n-6}{n^{5}} \sum_{i=1}^{n}\left\{h^{*}\left(X_{i}, X_{i}\right)\right\}^{2}+\frac{4\left(4 n^{2}-9 n+6\right)}{n^{5}(n-1)} \sum_{C_{n, 2}} \zeta_{2}\left(X_{i}, X_{j}\right) \\
+ & \frac{24(n-2)}{n^{5}} \sum_{C_{n, 2}} \frac{1}{2}\left\{h^{*}\left(X_{i}, X_{i}\right) h^{*}\left(X_{i}, X_{j}\right)+h^{*}\left(X_{i}, X_{j}\right) h^{*}\left(X_{j}, X_{j}\right)\right\} \\
+ & \frac{2(n-2)(n-3)}{n^{5}(n-1)} \sum_{C_{n, 2}} h^{*}\left(X_{i}, X_{i}\right) h^{*}\left(X_{j}, X_{j}\right) \\
+ & \frac{24(n-2)(2 n-3)}{n^{5}(n-1)} \sum_{C_{n, 3}} \zeta_{1}\left(X_{i}, X_{j}, X_{k}\right) \\
+ & \frac{12(n-2)(n-3)}{n^{5}(n-1)} \sum_{C_{n, 3}} \frac{1}{3}\left\{h^{*}\left(X_{i}, X_{i}\right) h^{*}\left(X_{j}, X_{k}\right)+h^{*}\left(X_{j}, X_{j}\right) h^{*}\left(X_{i}, X_{k}\right)\right. \\
+ & \frac{24(n-2)(n-3)}{n^{5}(n-1)} \sum_{C_{n, 4}} \zeta_{0}\left(X_{i}, X_{j}, X_{k}, X_{\ell}\right) .
\end{aligned}
$$

For the second term of (12), we have

$$
\left[\iint h^{*}\left(y_{1}, y_{2}\right) d F_{n}\left(y_{1}\right) d F_{n}\left(y_{2}\right)\right]^{2}=\frac{1}{n^{4}} \sum_{i=1}^{n} \sum_{j=1}^{n} \sum_{k=1}^{n} \sum_{\ell=1}^{n} h^{*}\left(X_{i}, X_{j}\right) h^{*}\left(X_{k}, X_{\ell}\right) .
$$


Thus we can show that

$$
\begin{aligned}
& {\left[\iint h^{*}\left(y_{1}, y_{2}\right) d F_{n}\left(y_{1}\right) d F_{n}\left(y_{2}\right)\right]^{2} } \\
= & \frac{1}{n^{4}}\left[\sum_{i=1}^{n}\left\{h^{*}\left(X_{i}, X_{i}\right)\right\}^{2}+4 \sum_{C_{n, 2}} \zeta_{2}\left(X_{i}, X_{j}\right)\right. \\
+ & 8 \sum_{C_{n, 2}} \frac{1}{2}\left\{h^{*}\left(X_{i}, X_{i}\right) h^{*}\left(X_{i}, X_{j}\right)+h^{*}\left(X_{i}, X_{j}\right) h^{*}\left(X_{j}, X_{j}\right)\right\} \\
+ & 2 \sum_{C_{n, 2}} h^{*}\left(X_{i}, X_{i}\right) h^{*}\left(X_{j}, X_{j}\right) \\
+ & 24 \sum_{C_{n, 3}} \zeta_{1}\left(X_{i}, X_{j}, X_{k}\right) \\
+ & 12 \sum_{C_{n, 3}} \frac{1}{3}\left\{h^{*}\left(X_{i}, X_{i}\right) h^{*}\left(X_{j}, X_{k}\right)+h^{*}\left(X_{j}, X_{j}\right) h^{*}\left(X_{i}, X_{k}\right)\right. \\
& \left.+h^{*}\left(X_{k}, X_{k}\right) h^{*}\left(X_{i}, X_{j}\right)\right\} \\
+ & \left.24 \sum_{C_{n, 4}} \zeta_{0}\left(X_{i}, X_{j}, X_{k}, X_{\ell}\right)\right] .
\end{aligned}
$$

From the above calculations, we get

$$
\begin{aligned}
\hat{\sigma}_{B}^{2}= & \iint\left(\begin{array}{l}
n \\
2
\end{array}\right)^{-2}\left[\sum_{C_{n, 2}} h^{*}\left(x_{i}, x_{j}\right)\right]^{2} \prod_{k=1}^{n} d F_{n}\left(x_{k}\right)-\left[\iint h^{*}\left(y_{1}, y_{2}\right) d F_{n}\left(y_{1}\right) d F_{n}\left(y_{2}\right)\right]^{2} \\
= & \frac{6(n-1)}{n^{5}} \sum_{i=1}^{n}\left\{h^{*}\left(X_{i}, X_{i}\right)\right\}^{2}+\frac{4\left(3 n^{2}-8 n+6\right)}{n^{5}(n-1)} \sum_{C_{n, 2}} \zeta_{2}\left(X_{i}, X_{j}\right) \\
& +\frac{16(n-3)}{n^{5}} \sum_{C_{n, 2}} \frac{1}{2}\left\{h^{*}\left(X_{i}, X_{i}\right) h^{*}\left(X_{i}, X_{j}\right)+h^{*}\left(X_{i}, X_{j}\right) h^{*}\left(X_{j}, X_{j}\right)\right\} \\
& -\frac{4(2 n-3)}{n^{5}} \sum_{C_{n, 2}} h^{*}\left(X_{i}, X_{i}\right) h^{*}\left(X_{j}, X_{j}\right)+\frac{24\left(n^{2}-6 n+6\right)}{n^{5}(n-1)} \sum_{C_{n, 3}} \zeta_{1}\left(X_{i}, X_{j}, X_{k}\right) \\
& -\frac{24(2 n-3)}{n^{5}(n-1)} \sum_{C_{n, 3}} \frac{1}{3}\left\{h^{*}\left(X_{i}, X_{i}\right) h^{*}\left(X_{j}, X_{k}\right)+h^{*}\left(X_{j}, X_{j}\right) h^{*}\left(X_{i}, X_{k}\right)\right. \\
& -\frac{48(2 n-3)}{n^{5}(n-1)} \sum_{C_{n, 4}} \zeta_{0}\left(X_{i}, X_{j}, X_{k}, X_{\ell}\right) .
\end{aligned}
$$

Using the $H$-decomposition, we will obtain asymptotic representation of $V_{B}=n \hat{\sigma}_{B}^{2}$ 
where

$$
\begin{aligned}
V_{B}= & \frac{6(n-1)}{n^{4}} \sum_{i=1}^{n}\left\{h^{*}\left(X_{i}, X_{i}\right)\right\}^{2}+\frac{4\left(3 n^{2}-8 n+6\right)}{n^{4}(n-1)} \sum_{C_{n, 2}} \zeta_{2}\left(X_{i}, X_{j}\right) \\
& +\frac{16(n-3)}{n^{4}} \sum_{C_{n, 2}} \frac{1}{2}\left\{h^{*}\left(X_{i}, X_{i}\right) h^{*}\left(X_{i}, X_{j}\right)+h^{*}\left(X_{i}, X_{j}\right) h^{*}\left(X_{j}, X_{j}\right)\right\} \\
& -\frac{4(2 n-3)}{n^{4}} \sum_{C_{n, 2}} h^{*}\left(X_{i}, X_{i}\right) h^{*}\left(X_{j}, X_{j}\right)+\frac{24\left(n^{2}-6 n+6\right)}{n^{4}(n-1)} \sum_{C_{n, 3}} \zeta_{1}\left(X_{i}, X_{j}, X_{k}\right) \\
& -\frac{24(2 n-3)}{n^{4}(n-1)} \sum_{C_{n, 3}} \frac{1}{3}\left\{h^{*}\left(X_{i}, X_{i}\right) h^{*}\left(X_{j}, X_{k}\right)+h^{*}\left(X_{j}, X_{j}\right) h^{*}\left(X_{i}, X_{k}\right)\right. \\
& \left.+h^{*}\left(X_{k}, X_{k}\right) h^{*}\left(X_{i}, X_{j}\right)\right\} \\
& -\frac{48(2 n-3)}{n^{4}(n-1)} \sum_{C_{n, 4}} \zeta_{0}\left(X_{i}, X_{j}, X_{k}, X_{\ell}\right) .
\end{aligned}
$$

For $x \neq y$, we have

$$
h^{*}(x, y)=g_{2}(x, y)+g_{1}(x)+g_{1}(y) .
$$

Let us obtain the $H$-decomposition for $\zeta_{2}$. It is easy to see that

$$
\begin{aligned}
& E\left[\zeta_{2}\left(x, X_{2}\right)\right] \\
= & E\left[g_{2}^{2}\left(x, X_{2}\right)+2 g_{2}\left(x, X_{2}\right) g_{1}\left(X_{2}\right)\right]+g_{1}^{2}(x)+\xi_{1}^{2}
\end{aligned}
$$

and

$$
\begin{aligned}
& E\left[\zeta_{2}(x, y)\right] \\
= & g_{2}^{2}(x, y)+2 g_{2}(x, y)\left\{g_{1}(x)+g_{1}(x)\right\}+g_{1}^{2}(x)+g_{1}^{2}(y)+2 g_{1}(x) g_{1}(y) .
\end{aligned}
$$

Since $E\left[\zeta_{2}\left(X_{1}, X_{2}\right)\right]=2 \xi_{1}^{2}+\xi_{2}^{2}$, we have the decompositions $\tilde{g}_{1}$ and $\tilde{g}_{2}$ of $\zeta_{2}$ where

$$
\begin{aligned}
\tilde{g}_{1}(x) & =E\left[\zeta_{2}\left(x, X_{2}\right)\right]-2 \xi_{1}^{2}-\xi_{2}^{2} \\
& =g_{1}^{2}(x)-2 \xi_{1}^{2}-\xi_{2}^{2}+E\left[g_{2}^{2}\left(x, X_{2}\right)+2 g_{2}\left(x, X_{2}\right) g_{1}\left(X_{2}\right)\right]
\end{aligned}
$$

and

$$
\begin{aligned}
\tilde{g}_{2}(x, y)= & E\left[\zeta_{2}(x, y)\right]-2 \xi_{1}^{2}-\xi_{2}^{2}-\tilde{g}_{1}(x)-\tilde{g}_{1}(y) \\
= & g_{2}^{2}(x, y)+2 g_{2}(x, y)\left\{g_{1}(x)+g_{1}(x)\right\}+2 g_{1}(x) g_{1}(y)+\xi_{2}^{2} \\
& -E\left[g_{2}^{2}\left(x, X_{2}\right)+g_{2}^{2}\left(y, X_{2}\right)+\left\{2 g_{2}\left(x, X_{2}\right)+2 g_{2}\left(y, X_{2}\right)\right\} g_{1}\left(X_{2}\right)\right] .
\end{aligned}
$$

Thus we can obtain the $H$-decomposition

$$
\begin{aligned}
& \left(\begin{array}{l}
n \\
2
\end{array}\right)^{-1} \sum_{C_{n, 2}} \zeta_{2}\left(X_{i}, X_{j}\right) \\
= & E\left[\left(\begin{array}{l}
n \\
2
\end{array}\right)^{-1} \sum_{k=1}^{2} \zeta_{2}\left(X_{i}, X_{j}\right)\right]+\left(\begin{array}{l}
n \\
2
\end{array}\right)^{-1} \sum_{C_{n, 2}}\left(\begin{array}{l}
n-k \\
2-k
\end{array}\right)^{2} A_{k} \\
= & 2 \xi_{1}^{2}+\xi_{2}^{2}+\frac{2}{n} \sum_{i=1}^{n} \tilde{g}_{1}\left(X_{i}\right)+\frac{2}{n(n-1)} \sum_{C_{n, 2}} \tilde{g}_{2}\left(X_{i}, X_{j}\right)
\end{aligned}
$$


Similarly we can obtain $H$-decomposition of $\zeta_{1}, \zeta_{0}$ as follows. It is easy to see that

$$
\begin{aligned}
& E\left[\zeta_{1}\left(x, X_{2}, X_{3}\right)\right] \\
= & \frac{2}{3}\left\{E\left[g_{2}\left(x, X_{3}\right) g_{1}\left(X_{3}\right)\right]+\xi_{1}^{2}\right\}+\frac{1}{3} g_{1}^{2}(x), \\
& E\left[\zeta_{1}\left(x, y, X_{3}\right)\right] \\
= & \frac{1}{3}\left\{g_{2}(x, y)\left\{g_{1}(x)+g_{1}(y)\right\}+g_{1}^{2}(x)+g_{1}^{2}(x)+3 g_{1}(x) g_{1}(y)+\xi_{1}^{2}\right. \\
& \left.+E\left[g_{2}\left(x, X_{3}\right) g_{2}\left(x, X_{3}\right)+\left\{g_{2}\left(x, X_{3}\right)+g_{2}\left(y, X_{3}\right)\right\} g_{1}\left(X_{3}\right)\right]\right\}
\end{aligned}
$$

and

$$
\begin{aligned}
& E\left[\zeta_{1}(x, y, z)\right] \\
= & \frac{1}{3}\left\{g_{2}(x, y) g_{2}(x, z)+g_{2}(x, y) g_{2}(y, z)+g_{2}(x, z) g_{2}(y, z)\right. \\
& +g_{2}(x, y)\left\{g_{1}(x)+g_{1}(y)+2 g_{1}(z)\right\}+g_{2}(x, z)\left\{g_{1}(x)+2 g_{1}(y)+g_{1}(z)\right\} \\
& +g_{2}(y, z)\left\{2 g_{1}(x)+g_{1}(y)+g_{1}(z)\right\}+g_{1}^{2}(x)+g_{1}^{2}(y)+g_{1}^{2}(z) \\
& \left.+3\left\{g_{1}(x) g_{1}(y)+g_{1}(x) g_{1}(z)+g_{1}(y) g_{1}(z)\right\}\right\} .
\end{aligned}
$$

Since $E\left[\zeta_{1}\left(X_{1}, X_{2}, X_{3}\right)\right]=\xi_{1}^{2}$, we have the decompositions $\hat{g}_{1}, \hat{g}_{2}$ and $\hat{g}_{3}$ of $\zeta_{1}$ where

$$
\begin{aligned}
\hat{g}_{1}(x)= & E\left[\zeta_{1}\left(x, X_{2}, X_{3}\right)\right]-\xi_{1}^{2} \\
= & \frac{1}{3}\left\{g_{1}^{2}(x)-\xi_{1}^{2}\right\}+\frac{2}{3} E\left[g_{2}\left(x, X_{2}\right) g_{1}\left(X_{2}\right)\right], \\
\hat{g}_{2}(x, y)= & E\left[\zeta_{1}\left(x, y, X_{3}\right)\right]-\xi_{1}^{2}-\hat{g}_{1}(x)-\hat{g}_{1}(y) \\
= & \frac{1}{3}\left\{g_{2}(x, y)\left\{g_{1}(x)+g_{1}(y)\right\}+3 g_{1}(x) g_{1}(y)\right. \\
& \left.+E\left[g_{2}\left(x, X_{3}\right) g_{2}\left(y, X_{3}\right)-\left\{g_{2}\left(x, X_{3}\right)+g_{2}\left(y, X_{3}\right)\right\} g_{1}\left(X_{3}\right)\right]\right\}
\end{aligned}
$$

and

$$
\begin{aligned}
\hat{g}_{3}(x, y, z)= & E\left[\zeta_{1}(x, y, z)\right]-\xi_{1}^{2}-\hat{g}_{2}(x, y)-\hat{g}_{2}(x, z)-\hat{g}_{2}(y, z) \\
& -\hat{g}_{1}(x)-\hat{g}_{1}(y)-\hat{g}_{1}(z) \\
= & \frac{1}{3}\left\{g_{2}(x, y) g_{2}(x, z)+g_{2}(x, y) g_{2}(y, z)+g_{2}(x, z) g_{2}(y, z)\right. \\
& +2\left(g_{2}(x, y) g_{1}(z)+g_{2}(x, z) g_{1}(y)+g_{2}(y, z) g_{1}(x)\right) \\
& \left.-E\left[g_{2}\left(x, X_{3}\right) g_{2}\left(y, X_{3}\right)+g_{2}\left(x, X_{3}\right) g_{2}\left(z, X_{3}\right)+g_{2}\left(y, X_{3}\right) g_{2}\left(z, X_{3}\right)\right]\right\} .
\end{aligned}
$$

Thus we get the $H$-decomposition

$$
\begin{aligned}
\left(\begin{array}{l}
n \\
3
\end{array}\right)^{-1} \sum_{C_{n, 3}} \zeta_{1}\left(X_{i}, X_{j}, X_{k}\right)= & \xi_{1}^{2}+\frac{3}{n} \sum_{i=1}^{n} \hat{g}_{1}\left(X_{i}\right)+\frac{6}{n(n-1)} \sum_{C_{n, 2}} \hat{g}_{2}\left(X_{i}, X_{j}\right) \\
& +\frac{6}{n(n-1)(n-2)} \sum_{C_{n, 3}} \hat{g}_{3}\left(X_{i}, X_{j}, X_{k}\right) .
\end{aligned}
$$


Furthermore, we can get the decompositions $\check{g}_{1}, \check{g}_{2}, \check{g}_{3}$ and $\check{g}_{4}$ of $\zeta_{0}$ as follows.

$$
\begin{aligned}
E\left[\zeta_{0}\left(X_{1}, X_{2}, X_{3}, X_{4}\right)\right]= & E\left[\zeta_{0}\left(x, X_{2}, X_{3}, X_{4}\right)\right]=\check{g}_{1}(x)=0, \\
E\left[\zeta_{0}\left(x, y, X_{3}, X_{4}\right)\right]= & \frac{2}{3} g_{1}(x) g_{1}(y), \\
E\left[\zeta_{0}\left(x, y, z, X_{4}\right)\right]= & \frac{1}{3}\left[g_{2}(x, y) g_{1}(z)+g_{2}(x, z) g_{1}(y)+g_{2}(y, z) g_{1}(x)\right. \\
& \left.+2\left\{g_{1}(x) g_{1}(y)+g_{1}(x) g_{1}(z) g_{1}(y) g_{1}(z)\right\}\right], \\
\check{g}_{2}(x, y)= & \frac{2}{3} g_{1}(x) g_{1}(y), \\
\check{g}_{3}(x, y, z)= & \frac{1}{3}\left\{g_{2}(x, y) g_{1}(z)+g_{2}(x, z) g_{1}(y)+g_{2}(y, z) g_{1}(x)\right\}
\end{aligned}
$$

and

$$
\check{g}_{4}(x, y, z, w)=\frac{1}{3}\left\{g_{2}(x, y) g_{2}(z, w)+g_{2}(x, z) g_{2}(y, w)+g_{2}(x, w) g_{2}(y, z)\right\} .
$$

Thus we have the $H$-decomposition for $\zeta_{0}$

$$
\begin{aligned}
& \left(\begin{array}{c}
n \\
4
\end{array}\right)^{-1} \sum_{C_{n, 4}} \zeta_{0}\left(X_{i}, X_{j}, X_{k}, X_{l}\right) \\
= & \frac{12}{n(n-1)} \sum_{C_{n, 2}} \check{g}_{2}\left(X_{i}, X_{j}\right)+\frac{24}{n(n-1)(n-2)} \sum_{C_{n, 3}} \check{g}_{3}\left(X_{i}, X_{j}, X_{k}\right) \\
& +\frac{24}{n(n-1)(n-2)(n-3)} \sum_{C_{n, 4}} \check{g}_{4}\left(X_{i}, X_{j}, X_{k}, X_{l}\right) .
\end{aligned}
$$

Combining the equations (13), (14), (15) and moment evaluations, we get the desired result (8).

\section{Acknowledgement}

This research was supported by JSPS Grant-in-Aid for Scientific Research (B) No.21340026 and Exploratory Research No.21650065.

\section{References}

Dharmadhikari, S.W., Fabian, V. and Jogdeo, K. (1968). Bounds on the moments of martingales. Ann. Math. Statist., 39, 1719-1723.

Efron, B. and Stein, C. (1981). he jackknife estimate of variance. Ann. Statist., 9, 586596.

Hinkley, D.V. (1978). mproving the jackknife with special reference to correlation estimation, Biometrika. 65, 13-21.

Hoeffding, W. (1961). he strong law of large numbers for $U$-statistics, Univ. of North Carolina Institute of statistics. Mimeo Series. No.302. 
Maesono, Y. (1998). Mean square errors of variance estimators and their Edgeworth expansions. Journal of the Japan Statistical Society, 28, 1-19.

Petrov, V.V. (1975). Sums of Independent Random Variables, Springer Berlin.

Schucany, W.R. and Bankson, D.M. (1989). Small sample variance estimators for $U$ statistics. Austral. J. Statist. 31, 417-426.

Sen, P.K. (1960). n some convergence properties of $U$-statistics. Calcutta Statist. Assoc. Bull., 10, 1-18.

Shirahata, S. and Sakamoto, Y. (1992). Estimate of variance of $U$-statistics. Commun. Statist.- Theory Meth., 21, 2969-2981.

von Bahr, B and Esséen C.G. (1965). Inequalities for the $r$ th absolute moment of a sum of random variables, $1 \leq r \leq 2$. Ann. Math. Statist., 36, 299303.

Received August 25, 2011

Revised November 20, 2011 